\title{
The observational signature of quasi period reconnection
}

\author{
G. P. Wu $\mathbf{u}^{1,2} \dagger$ and G. L. Huang ${ }^{1}$ \\ Purple Mountain Observatory, Chinese Academy of Sciences, Nanjing 210008, China \\ email:wuguiping@seu.edu.cn \\ Department of Physics, Southeast University, Nanjing 210096, China
}

\begin{abstract}
Based upon the similar time profiles of the hard x-ray and microwave emissions on 21 April 2002 flare-CME event, we argue that: (1) in the impulsive phase of the flare, the fast looploop reconnection triggered the quasi periodic magnetic energy release and particle acceleration with duration of the order of minute, $(2)$ in the extended phase of the flare (01:40-01:50UT), the quasi period reconnection in current sheet produced the impulsive energetic electrons injection and modulated the microwave emissions with duration of 10-30 s, (3) the current driven instability enhances the magnetic energy releasing rate and scatters the energetic electrons, which may be used to explain the power index of the hard X-ray and microwave emissions.
\end{abstract}

Keywords. quasi periodic oscillation, fast loop-loop reconnection, current driven instability

\section{Observation}

There are many time scales in the hard x-ray and microwave emissions of the M1.5 flare at S14 W84 on 21 April 2002, which may tell us the physical nature related to the magnetic energy release and the particle acceleration.

During the impulsive phase of the flare, the similar quasi periodic oscillation modulated the hard x-ray and radio emissions with duration of about 1-3 minutes ( seen in figure 1). The newly emerged small loop contacted the upper large loop and these loops became bright gradually from trance $195 \AA$ image. While in the extended phase (01:40-01:50UT), as shown in the figures 2 and 3 of Huang and Lin (2006), the quasi periodic oscillations (10-30 s) in Stokes I and V of $17 \mathrm{GHz}$ was found and the loop top source in $17 \mathrm{GHz}$ rose in the speed of about $10 \mathrm{~km} \mathrm{~s}^{-1}$, which may result from the impulsive particle acceleration and injection. In addition, previous studies on the same flare showed that there was a rapidly expending fan like a current sheet, the energy releasing process was in agreement with the classic flare model, and the $12-25 \mathrm{keV}$ source centroid moves away from the limb with a apparent velocity of $9.9 \mathrm{~km} \mathrm{~s}^{-1}$ (Gallagher et al., 2002).

\section{Discussion}

As we know, the fast loop-loop reconnection may be modulated with the quasi period of the order of minute ( $\mathrm{Wu}$ et al., 2005a and references therein). The induced electric field may accelerate electrons with the characteristics of quasi periodic oscillation, which result in the common modulation in the hard x-ray and microwave emissions. So, from the above observations in the impulsive phase of the flare, we argue that the loop-loop interaction triggered the magnetic energy release and accelerated the electrons.

$\dagger$ Present address: 2nd, Si Pai-Lou, Department of Physics, Nanjing, 2100096, China. 

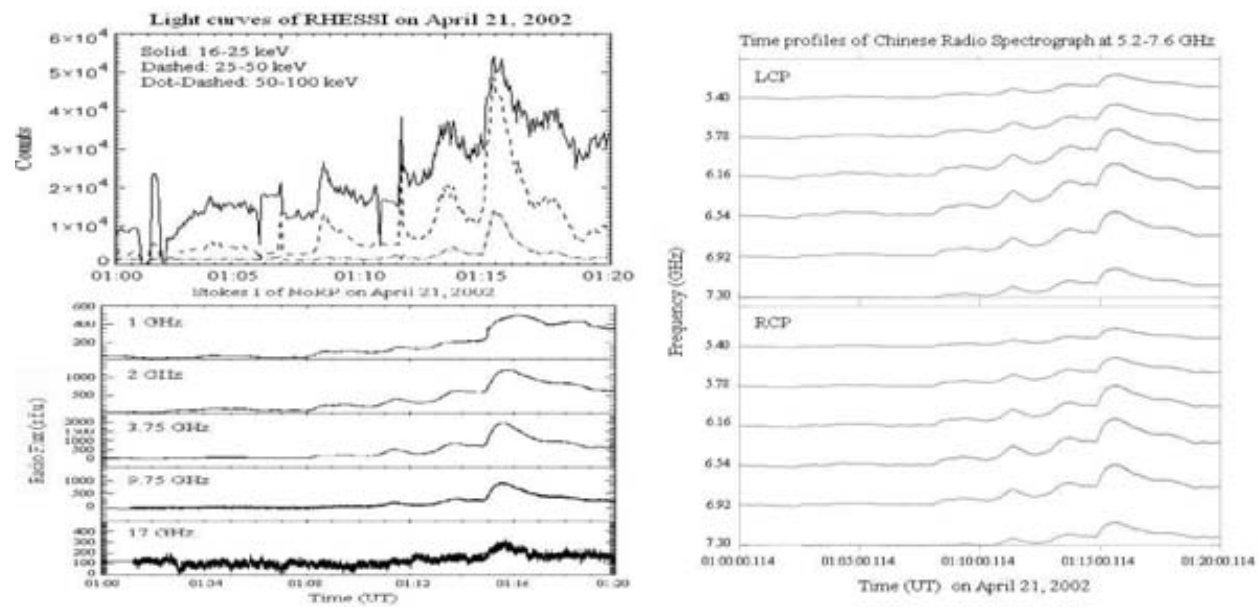

Figure 1. RHESS light curves, the Stokes I profiles at 1, 2, 3.75, 9.75 and $17 \mathrm{GHz}$ of Nobeyama Radio Heliograph (NoRP) and the profiles of Chinese Radio Spectrograph at $5.2-7.6 \mathrm{GHz}$ for 01:00-01:20 UT.

As the current sheet ascended during the extended phase of the flare, the induced electric field strength in the reconnecting current sheet(RCS) is decided by the inflow velocity and the local magnetic field strength. The typical acceleration time of energetic electrons inside RCS is about $10^{-6} \sim 10^{-5} \mathrm{~s}$, which is much less than the oscillation period (Wu et al. 2005b). So, we may argue that the time profile of Stokes I in $17 \mathrm{GHz}$ may reveal the change of the induced electric field and further the inflow velocity, which means that reconnection is quasi period. After considering the heat conduction and the anomalous resistivity in their magnetic reconnection simulation, Chen et al. (1999) found the similar oscillation with the period of $2 \sim 4 L / v_{A}$ (here $\mathrm{v}_{A}$ the Alfven velocity and $L$ the sheet length). With the typical value of $\mathrm{B}=100 \mathrm{Gs}, L=10^{9} \mathrm{~cm}$ and the plasma density of $10^{10} \mathrm{~cm}^{-3}$, the oscillation period is about $10 \sim 20 \mathrm{~s}$. Therefore, we may infer that as the plasma is heated, the thermal pressure increases and the inflow velocity decreases, when the heat conduction causes the plasma temperature inside RCS decreases, the inflow velocity increases. Thus, the quasi period reconnection is formed.

As inferred from the microwave emissions, the power-law index of the nonthermal electrons is about $4 \sim 5$ in the extended phase (Huang and Lin, 2006). It is too soft to be interpreted in the theoretic model of the particle acceleration inside the collisionless RCS without considering the wave-particle interaction. After taking these effects into consideration, we got a power-law distribution of energetic electrons with index of $3 \sim 10$ through solving Vlasov equation in the simplified model, which may be used to explain the observations ( $\mathrm{Wu}$ et al. 2005b).

\section{Acknowledgements}

This study is supported by the NFSC projects with No.10273025 and 10333030 .

\section{References}

P. Chen, C. Fang, Y. Tang, and M. Ding, 1999, ApJ, 513, 516

Gallagher, P. T., Dennis, B. R., Krucker, S. et al., 2002, Solar Phys., 210, 341

G. Huang and J. Lin 2006, ApJ, 639, L99

G. Wu, G. Huang, Y. Tang and Xu, A. A., 2005a, Solar Phys, 227, 327

G. Wu, G. Huang, and Y. Tang, 2005b, ChJAA, 5, 99 